\title{
Die Zeiten ändern sich. Zur Verwendung des imparfait in narrativen Kontexten
}

\author{
Von Angela Schrott
}

\begin{abstract}
Using the imparfait in narrative contexts seems to contradict the imperfective aspect of the form, as the perfective aspect seems far more adequate in these contexts. In order to understand the effects of this usage, the imparfait narratif is considered on two levels: on the linguistic level of the French language and its aspect system, and on a text level that embraces the cultural discourse traditions that characterise different text types and genres. On the linguistic level, the imparfait is first contrasted to its well-known perfective counterpart, the passé simple. In a second step, the article brings into view the analogy between the présent and the imparfait from a pragmalinguistic perspective: both forms share the inherent reference to an anchor-situation that encompasses a temporal as well as a personal dimension. On the text level, two usage-types of the imparfait in narrative contexts can be distinguished. Whereas the first type emphasises the temporal dimension of the anchor-situation, the second type focuses on the personal dimension and is closely linked to narrative techniques of point of view. As the analysis shows, both types result from the interaction between linguistic form and cultural discourse traditions. In conclusion, the use of the imparfait in narrative contexts in fact indicates a change. However, this change does not take place on the linguistic level of the aspect system but in the realm of the discourse traditions of narration that guide the use of the imparfait in texts.
\end{abstract}

Eine Verwendung des imparfait, die in der Forschung mehrfach als Indiz für eine Veränderung im temporal-aspektuellen System des Französischen gedeutet wurde, ist dessen Gebrauch in narrativen Kontexten. In diesen Fällen versprachlicht das imperfektive imparfait Handlungen und Ereignisse, die eine Sukzession in der Zeit abbilden und für deren Ausdruck eine perfektiv markierte Form erwartbar ist. Diese der aspektuellen Markierung zunächst widersprechende Verwendung als imparfait narratif ist der Ausgangspunkt für die Frage, ob und inwiefern sich das Profil des imparfait im Französischen verändert ${ }^{1}$.

Grundsätzlich kann die Verwendung des imparfait in narrativen Kontexten auf zwei Ebenen untersucht werden: auf der Ebene des Französischen als historische Einzelsprache und auf der Ebene der Texte, in denen das imparfait verwendet

1 Ich widme diesen Aufsatz dem Andenken meines Lehrers und Freundes Claus Morgenstern (1925-2009). Seine Gedanken zu Tempus und Aspekt haben mich viele Jahre lang inspiriert und begleitet. 
wird. Im ersten Fall steht das Gefüge temporal-aspektueller Formen des Französischen im Zentrum, im zweiten Fall geht es um die Effekte, die das imparfait im Zusammenwirken mit anderen Mitteln der Textgestaltung in Texten erzeugt. Ausgangspunkt des hier gewählten Ansatzes ist, dass erst die Zusammenschau beider Ebenen es ermöglicht, das semantisch-pragmatische Profil des imparfait zu erschließen. Daher steht zunächst die imparfait-Bedeutung im Mittelpunkt, die kontrastiv zum passé simple, vor allem aber im Vergleich zum présent analysiert wird. Diese doppelte Bezugsetzung klärt und erklärt, welche Wirkungen das imparfait in narrativen Kontexten entfaltet und in welcher Weise sich die Traditionalität des Sprechens verändert. Die mit dem Typ des imparfait narratif verbundene Frage nach Stabilität und Wandel des imparfait ist ein geeignetes Feld, um die Dynamik des semantisch-pragmatischen Profils von Aspektformen auch sprachtheoretisch hinsichtlich der in die Sprachverwendung eingehenden Wissensbestände und Traditionen zu charakterisieren.

\section{Einzelsprachliche Bedeutung und Textsemantik}

Für das Wirken des imparfait in Texten ist die Relation von einzelsprachlicher Bedeutung und Textsemantik entscheidend. Grundlegend ist hier das Verständnis der in den Text eingehenden Wissensbestände. Ausgehend vom Modell der Sprachkompetenz nach Eugenio Coseriu werden kategoriell drei Wissensbestände angesetzt: das Wissen um allgemeine Prinzipien und Regeln des Sprechens, das einzelsprachliche Wissen und das kulturelle diskurstraditionelle Wissen ${ }^{2}$. Die Diskurstraditionen sind dabei derjenige Wissensbestand, der die Selegierung sprachlicher Elemente und deren Arrangement in Texten anleitet und damit den Text als Repräsentant einer Textgattung gestaltet und modelliert.

Zunächst zur Semantik der imparfait-Form. Grundsätzlich ist das französische Verbalsystem durch die Besonderheit charakterisiert, dass die Verbalformen sowohl als Tempus als auch als grammatischer Aspekt funktionieren ${ }^{3}$. Während Tempora eine deiktische Situierung in Bezug zur Sprechsituation leisten, modellieren Aspekte den zeitlichen Verlauf des versprachlichten Sachverhalts, ohne eine deiktische Situierung relativ zum ego-hic-nunc zu etablieren. Auch das imparfait erfüllt daher eine temporaldeiktische Funktion und fungiert zugleich als grammatischer Aspekt, sodass die imparfait-Bedeutung temporaldeiktische und aspektuelle Semantik vereint. Der Aspekt ist dabei kein Effekt der temporaldeiktischen Funktion, sondern stellt eine eigene verbale Kategorie dar. Bei der Verwendung des imparfait (oder einer anderen Verbalform) in Texten geht diese temporal-aspektuelle Bedeutung als einzelsprachlicher Wissensbestand in die Textsemantik ein und ergibt in Wechselwirkung mit den anderen im Text präsenten Wissensbeständen

2 Zur Sprachkompetenz und ihren Wissensbeständen vgl. Coseriu (1988: 70-75; 2000: 88 ff.), Schrott (2006) und Schrott (2011: 194-196).

3 Zum romanischen Verbalsystem und seiner Kombination der Kategorien Tempus und Aspekt vgl. etwa Coseriu (1976: 81 f.) und Bres (2005b: 5 f.). 
dann den der Ebene der Texte angehörenden Textsinn als eine Größe, die sich übersummativ aus sämtlichen in den Text eingehenden Wissensbeständen zusammensetzt ${ }^{4}$. Die Funktion des imparfait in Texten erschließt sich damit erst in einem textsemantischen Zugriff.

Grundlegend für das Zusammenspiel zwischen imparfait-Semantik und Kontext ist ferner die Feststellung, dass das imparfait als Aspektform ${ }^{5}$ mit verschiedenen Textelementen und Charakteristika des Textes in unterschiedlicher Weise interagiert. So sind etwa temporale Angaben Elemente, die mit dem imparfait eng zusammenwirken und oft in einer Beziehung der Affinität stehen ${ }^{6}$. Betrachtet man das Zusammenwirken von Verbalformen und temporalen Angaben, dann fällt auf, dass temporale Angaben Elemente sind, die im Text eingrenzbar sind und auf der Fläche des Textes gleichsam ,,punktuell“ auftreten. Um das imparfait zu charakterisieren, ist daher eine in der aktuellen Forschung zur Textsemantik getroffene Unterscheidung nützlich: die Differenzierung zwischen punktuellen und flächigen Phänomenen ${ }^{7}$. Übertragen auf das Wirken von Aspektformen in Texten meint die punktuelle Interaktion die Wechselwirkung des imparfait mit begrenzten und begrenzbaren Elementen im Text, etwa mit temporalen Angaben oder auch mit Verben und deren Aktanten. Eine flächige Interaktion dagegen liegt vor, wenn die imparfait-Semantik mit einem Parameter interagiert, der den Text in seiner Gesamtheit prägt und nicht an punktuell auftretenden Elementen festgemacht werden kann. Ein solches flächiges Phänomen ist etwa das Erzählen als eine den Text als Ganzes prägende Technik. Für die Charakterisierung des imparfait in narrativen Kontexten spielen sowohl punktuelle als auch flächige Phänomene und Formen der Interaktion eine Rolle.

${ }^{4}$ Der Begriff des Textsinns folgt hier dem Konzept des Sinns nach Coseriu (1988: 87-89). Zum Modell einer als ,aktuell-textuelle Bedeutung“ konzipierten Textsemantik vgl. Gardt (2002: bes. $121 \mathrm{f}$., 124, $128 \mathrm{f}$.).

5 Da für das Funktionieren des imparfait in narrativen Kontexten die Aspektmarkierung grundlegend ist, wird das imparfait im Folgenden als Aspektform geführt. Auf die ebenfalls gegebene temporaldeiktische Funktion wird nicht mehr gesondert hingewiesen.

6 Tempora und temporale Angaben werden daher in der Forschung mehrfach als ein zusammengehöriges System analysiert, vgl. etwa Klum (1961) und Vet (1980). In vielen Fällen favorisieren oder bedingen temporale Angaben bestimmte temporal-aspektuelle Formen. $\mathrm{Zu}$ diesen Affinitäten und ihrer Typisierung im Allgemeinen vgl. Schrott (1997: 337-343); zu temporalen Angaben beim imparfait vgl. Togeby (1982: 332-354).

7 Diese Differenzierung greift auf Gardt (2008: 214 f.) zurück, der im Bereich der Textsemantik „punktuelle“ und „flächige“ Phänomene unterscheidet. Während punktuelle Bedeutungsbildung an einzelnen, begrenzten Textstellen (wie z. B. lexikalischen Ausdrücken) ansetzt, vollzieht sich flächige Bedeutungsbildung in der Summe des Zusammenwirkens semantischer Textelemente (ebd.). Das Begriffspaar erfasst über die in Gardt (2008) behandelten Phänomene hinaus zwei grundlegende Kategorien der Konstituierung von Textsinn und hat daher auch für das Wirken von Tempus und Aspekt in Texten Gültigkeit. 


\section{Die einzelsprachliche Bedeutung des imparfait}

\subsection{Imperfektiver Aspekt und Lesarten der Imperfektivität}

Die Bedeutung des imparfait umfasst neben der temporaldeiktischen Semantik einer Situierung in der Vergangenheit die Semantik des imperfektiven Aspekts. Als Kategorie leisten grammatische Aspekte die Markierung der zeitlichen Verfasstheit und Konturiertheit von Sachverhalten: Ereignisse und Handlungen erhalten durch den Aspekt im Akt der Versprachlichung eine zeitliche Modellierung ${ }^{8}$. Der imperfektive Aspekt des imparfait beinhaltet, dass Sachverhalte unter Ausblendung ihrer Begrenzungen versprachlicht werden ${ }^{9}$. Anfang und Ende eines Sachverhalts werden beim imperfektiven Aspekt nicht in den Blick genommen, sodass die sprachliche Darstellung von in der außersprachlichen Wirklichkeit gegebenen Begrenzungen in der Zeit abstrahiert ${ }^{10}$. Die folgenden beiden Beispiele illustrieren diese Unbegrenztheit des imperfektiven Aspekts:

(1) Mon père, qui s'appelait Joseph, était alors un jeune homme brun, de taille médiocre, sans être petit. Il avait un nez assez important, mais parfaitement droit [...]. (Pagnol: Gloire, S. 21)

(2) Je retrouvai, sans aucune joie, la grande école: les platanes de la cour commençaient à perdre leurs feuilles jaunies [...]. (Pagnol: Château, S. 102)

In (1) beschreibt der Erzähler Statur und Physiognomie seines Vaters als Eigenschaften bzw. Sachverhalte, die in der evozierten Situation in der Vergangenheit (,alors") gegeben sind, ohne dass Anfang oder Ende thematisiert werden. Die gleiche Ausblendung zeitlicher Begrenzungen charakterisiert auch die imparfait-Verwendung in (2). Bei der Rückkehr in die Schule nach den Sommerferien ist das beginnende Fallen der Blätter ein Sachverhalt, der sich „schon und noch“ vollzieht. Die Beispiele dokumentieren, dass das imparfait als grammatischer Aspekt unterschiedlichen Typen von Sachverhalten eine imperfektive Prägung verleiht. So geht es in (1) um Sachverhalte, die als konstante Eigenschaften konzeptualisiert

8 Prägend für die Forschung ist Comries Definition der Aspektkategorie als ,internal temporal constituency“ eines Sachverhalts (Comrie 1976: 5, 24-26). Zur Aspektkategorie vgl. auch Fleischman (1982: 11), Maslov (1985: 3 f.) und Kleiber (2003: 9-12). Einen detaillierten und (nach wie vor) aussagekräftigen Forschungsüberblick gibt Pollak ( ${ }^{2} 1988$ : 20-82).

9 Bei der Betrachtung von Aspekt und Aspekttypen ist dabei zwischen einer übereinzelsprachlich-typologischen Beschreibung (Comrie 1976: 16-26, bes. 17-19), die nach Coseriu (1988) auf der allgemein-universellen Ebene des Sprechens zu verorten wäre, und einzelsprachlichen Charakterisierungen zu differenzieren, die der historisch-einzelsprachlichen Ebene angehören. Zum Aspekt in den romanischen Sprachen vgl. Coseriu (1976: 112-114, 139-144), Hunnius (2009: 54 f.) und Becker (2010a: bes. 83-85). Zum Aspekt im Französischen vgl. etwa Imbs (1960: 96 f.), Vet (1980: 76-81), Togeby (1982: 318 f.), Pollak (21988: bes. 117-124), Mitko (2000: 23-25, 83-103) und Barbazan (2006: 85-116).

10 Becker (2010a: 85) deutet diese Ausblendung der Grenzen semantisch als Unterdeterminiertheit des imparfait. 
sind und in aller Regel nicht unter dem Gesichtspunkt zeitlicher Begrenzung oder Veränderbarkeit gesehen werden. Beispiel (2) dagegen thematisiert den Sachverhalt des Beginnens, der dem Konzept einer Anfangsbegrenzung nahe steht, hier aber durch den imperfektiven Aspekt als „schon und noch“ gegebener, prozesshaftgedehnter Neueinsatz gesehen wird. Der von der Verbalform transportierte grammatische Aspekt fungiert damit als Stempel, der verschiedensten zeitlichen Konzepten und sprachlichen Bedeutungen aufgedrückt werden kann ${ }^{11}$.

Wie die Beispiele exemplarisch illustrieren, sind Sachverhalte im imparfait in einer Situation in der Vergangenheit ohne zeitliche Begrenzung gegeben und in diesem Sinne „unbegrenzt seiend“. Sie erscheinen damit als „präsentisch“ zu einer vergangenen Situation. Diese Situation ist zentral für das semantische Profil des imparfait. Denn ein Sachverhalt, dessen Ränder imperfektiv ausgeblendet werden und daher nicht als situierender Zeitanker fungieren können, benötigt als Verankerung immer eine Situation, in der er "schon und noch“ gegeben ist. Für diese Situation wird im Folgenden der Begriff „Ankersituation“ verwendet ${ }^{12}$. Ereignisse und Handlungen im imparfait haben damit die grundlegende Eigenschaft, dass sie in einer Situation in der Vergangenheit unter Ausblendung ihrer Begrenzungen präsentisch $\operatorname{sind}^{13}$. Diese Präsentischkeit manifestiert sich in drei unterschiedlichen Lesarten. Die erste Lesart veranschaulichen die Beispiele (1) und (2), in denen die im imparfait ausgedrückten Sachverhalte kontinuierlich gegeben sind. Eine zweite Variante besteht darin, dass das imparfait sich wiederholende Ereignisse ausdrückt:

(3) On fêtait un jour, solennellement, ses palmes académiques: trois ans plus tard, il „prenait sa retraite“, c'est à dire que le règlement la lui imposait. Alors, souriant de plaisir, il disait: „Je vais enfin pouvoir planter mes choux!“ Sur quoi il se couchait, et il mourait. J'en ai connu beaucoup, de ces maitres d'autrefois. (Pagnol: Gloire, S. 19)

11 Kognitive Konzepte, die Unveränderlichkeit implizieren, haben zwar Affinität zum Ausdruck durch den imperfektiven Aspekt, doch liegt hier keine Korrelation vor und auch konträre (perfektive) Aspektmarkierungen sind in spezifischen Kontexten möglich. Zur Interaktion von grammatischen Aspekten und Situationstypen und zur Diskussion von Affinitäten und (eventuellen) Restriktionen vgl. Mitko (2000: 140 f.) und Schrott (2003: $254 \mathrm{f}$.).

12 In der Forschung findet sich hier meist der Begriff der Referenzsituation, der aufgrund der Forschungstradition oft auf eine rein zeitliche Dimension begrenzt ist. Der hier gewählte (neue) Begriff der Ankersituation ist dagegen offener für nicht-zeitliche Dimensionen von Situationalität. Zum Konzept der Referenzsituation in der Tradition der ,approches référentielles“vgl. Molendijk (1985: bes. 78-81) und de Saussure / Sthioul (1999: 170-173).

13 Perfektiver und imperfektiver Aspekt werden auch durch die Metapher der Perspektive als Innensicht (imperfektiver Aspekt) vs. Außensicht (perfektiver Aspekt) erfasst (vgl. Mitko 2000: 23-25). Diese Spatialisierung ist eine Veranschaulichung abstrakt-zeitlicher Konturen, die jedoch nicht alle imparfait-Verwendungen erklären kann, da der Aspekt keine spatiale Kategorie ist; vgl. hierzu auch Schrott (2003: 251). Die imparfait-Bedeutung kann daher präziser über die Kriterien der unbegrenzten Präsentischkeit in einer Ankersituation erfasst werden. 
Geschildert wird nicht die individuelle Biographie eines instituteur, sondern ein Lebenslauf, wie er für die „maîtres d'autrefois“ charakteristisch ist: Der typische instituteur erhält die palmes académiques, geht drei Jahre später mit der Aussicht, nun endlich seinen Garten bestellen zu können, in den Ruhestand - und segnet kurz darauf das Zeitliche. Die Handlungssequenz wiederholt sich in diesem Fall als Gesamtheit unbegrenzt oft, sodass die Grenzen der sich wiederholenden Handlung imperfektiv ausgeblendet sind ${ }^{14}$. Da die iterierenden Handlungen mit Unterbrechungen in der Zeit realisiert werden, liegt in der Gesamtheit der sich wiederholenden Handlungen ein diskontinuierlicher Verlauf vor.

Zum kontinuierlichen Typ und zur Variante der diskontinuierlichen unbegrenzten Iteration kommt nun noch ein dritter Typ von Präsentischkeit hinzu:

(4) Quand Yvars arriva au vestiaire, Esposito [...] était déjà sous la douche. Il leur tournait le dos, tout en se savonnant à grand bruit. (Camus: Les muets, S. 100)

Anders als in den Beispielen (1) bis (3) ist in (4) in der geschilderten Situation nicht die Handlung an sich gegeben, sondern vielmehr die daraus resultierende Nachphase: Der Protagonist dreht sich nicht als Reaktion auf den ankommenden Yvars um, sondern steht bereits mit dem Rücken $\mathrm{zu}^{\mathrm{ihm}}{ }^{15}$. Das imparfait versprachlicht damit die in einer Ankersituation präsentische Nachphase einer Handlung, wobei die eigentliche Aktion gleichsam übersprungen wird.

Durch seine temporal-aspektuelle Bedeutung hat das imparfait das Potenzial, im Zusammenwirken mit unterschiedlichen Kontextelementen drei Lesarten der Präsentischkeit zu erzeugen. Diese Interaktion kann punktuell erfolgen, etwa wenn allein das Verb (wie bspw. s'appeler) in Beispiel (1) als punktgenau lokalisierbares Textelement eine kontinuierliche Lesart festsetzt. In Beispiel (3) dagegen klärt der abschließende Kommentar des Erzählers („J'en ai connu beaucoup, de ces maîtres d'autrefois“) die Iterativität der zuvor beschriebenen Ereignisse. Da dieser Kommentar dem Textabschnitt in seiner Ganzheit exemplarischen Charakter verleiht, hat die Wechselwirkung zwischen imparfait-Formen und Erzählerkommentar hier einen flächigen Charakter. Die drei Typen einer unbegrenzten Präsentischkeit sind damit Subtypen des imperfektiven Aspekts, die sich als textsemantisch erzeugte Funktionen der Form ergeben.

$14 \mathrm{Zu}$ iterativen Verwendungen vgl. etwa Togeby (1982: 319) und Becker (2010a: 98 f., 101). Haben die sich wiederholenden Handlungen habituellen Charakter, dann kann die unbegrenzte Iteration als auf Dauer gestellte Gewohnheit wiederum eine Kontinuität „höherer Ordnung" besitzen.

15 Das imparfait erfüllt hier eine Funktion, die der Semantik des plus-que-parfait verwandt ist. Ein Hinweis auf dieses Phänomen findet sich im Modell Coserius (1976: 148 f.): Da das imparfait eine retrospektive Komponente habe, könne es in bestimmten Fällen (als unmarkiertes Glied der Opposition) das plus-que-parfait vertreten. Vgl. zu dieser Verwendung auch Berthonneau (2000: 83). 


\subsection{Das imparfait und seine Ankersituation}

Für das Verständnis der imparfait-Verwendung in narrativen Kontexten ist das Konzept der Ankersituation zentral. Grundsätzlich kann die Ankersituation in Texten in unterschiedlichem Grade explizit bzw. implizit gegeben sein und muss entsprechend in mehr oder weniger hohem Grad inferentiell erschlossen werden. Dazu zwei Beispiele:

(5) Les écoles normales primaires étaient à cette époque de véritables séminaires, mais l'étude de la théologie y était remplacée par des cours d'anticléricalisme. (Pagnol: Gloire, S. 15)

(6) - „Eh bien, voilà trois francs de gaspillés!“

- „Mais ma chérie, si tu voulais fabriquer ce cor de chasse, pense à l'achat du cuivre, pense à l'outillage spécial qu'il te faudrait [...].“ Ma mère haussait doucement les épaules [...]. (Pagnol: Gloire, S. 58)

In (5) ist die Existenz der ,écoles normales primaires“ in einer Ankersituation präsentisch, die durch eine temporale Angabe (,à cette époque“) explizit im Text genannt ist. In (6) dagegen ist die Ankersituation für die Handlung der Mutter (,,ma mère haussait doucement les épaules") nur inferentiell erschließbar: Die Geste begleitet die Worte des Vaters als nonverbaler Kommentar, sodass die Ankersituation für das imparfait durch die Replik des Vaters geliefert wird. Anders als in (5) ist die Ankersituation in (6) dem Text implizit und muss erst erschlossen werden.

Die Beispiele illustrieren, dass die Ankersituation eine Größe ist, die im Kontext mehr oder weniger explizit enthalten ist bzw. die in einem unterschiedlichen Grade inferentiell zu erschließen ist. Eine grundlegende Frage ist nun, welchen Anteil die sprachliche Bedeutung des imparfait an der Konstituierung der Ankersituation hat. Die für das imparfait charakteristische Rückbindung an eine Situation begründet auch dessen Deutung als anaphorische Form, die ein (inferierbares) Antezedens benötigt ${ }^{16}$. Die entscheidende Innovation in der Diskussion um das anaphorische imparfait liefert das von Berthonneau / Kleiber (1993) formulierte Modell des imparfait als anaphorisch-meronymische Form, das die Beschränkung auf die rein zeitliche Dimension aufbricht und die imparfait-Semantik über eine Teil-Ganzes Beziehung definiert ${ }^{17}$. Der im imparfait versprachlichte Sachverhalt erscheint als Teil einer Situation, und diese Situation bildet wiederum das größere Ganze, deren Bestandteil die im imparfait ausgedrückte Handlung ist ${ }^{18}$.

16 Becker (2010a: 86, 92) beschreibt das imparfait (und seine Äquivalente in den romanischen Sprachen) daher als unterspezifizierte Formen, die lediglich eine partielle referentielle Autonomie besitzen, und unterstreicht so aus zeitreferentieller und tempuslogischer Sicht, dass das imparfait einen temporalen oder modalen „Anker“ benötigt.

17 Berthonneau / Kleiber setzen bei anaphorischen Ansätzen an (1993: 56-62), deren Erklärungsprobleme sie aufschlüsseln (1993: 63-66) und lösen, indem sie die bis dahin rein zeitliche Sicht der Konzepte Simultaneität bzw. Koreferenz durch eine meronymische Relation ersetzen (1993: 66-70, bes. 68). Vgl. hierzu auch Bres (2005b: 13 f.).

18 Das imparfait ist nach Berthonneau / Kleiber eine Form „qui introduit un nouvel événement ou état en le présentant comme une partie ou un ingrédient d'une situation passée 
Bereits im Modell von Berthonneau / Kleiber (1993) dominiert die meronymische Relation das anaphorische Konzept, doch bleibt im Modell Berthonneaus und Kleibers das Konzept der Anaphorizität erhalten, da die Situation im Ko(n)text gegeben oder inferierbar sein muss. Die hier unternommene Erklärung des imparfait über eine Ankersituation zielt nun auf eine noch weitergehende Stärkung der meronymischen Relation. Betrachtet man in der Meronymie Teil und Ganzes als zwei gleichberechtigte Teilkonzepte, dann setzt die Existenz des Teils immer schon das Ganze voraus. Als Teil eines größeren Ganzen präsupponiert das imparfait damit pars pro toto das Ganze und damit die Situation, deren Teil die versprachlichte Handlung ist. Auf diese Weise besitzt das imparfait unabhängig von kontextuellen Stützungen immer eine Ankersituation, die es in den Text einzubringen vermag. Eine so verstandene meronymische Semantik steht über das Modell der Ankersituation auch in Einklang mit der Deutung des imparfait als imperfektiver Aspekt. Denn auch die aspektuelle Semantik eines Sachverhalts, der in einer Situation „unbegrenzt seiend“ ist, beinhaltet durch den Bezug auf diese Situation das für die Meronymie konstitutive Konzept der Ankersituation. Imperfektiver Aspekt und Meronymie sind als Erklärungsmodelle daher zwei Seiten einer Medaille. Die das imparfait charakterisierende Formel „unbegrenzt präsentisch seiend zu einer Situation in der Vergangenheit" ist sowohl meronymisch als auch aspektuell deutbar. Beides sind operable Erklärungsmuster für die imparfaitKategorie, die die Beschränkung auf die reine Zeitlichkeit überwinden ${ }^{19}$. Aufschlussreich für diese Inhärenz der Ankersituation sind Textbelege, in denen das imparfait am Beginn eines Textes - etwa einer Erzählung - eingesetzt wird. Die folgenden Textausschnitte - es handelt sich um die Anfänge zweier Novellen von Camus - illustrieren solche Verwendungen:

(7) L'instituteur regardait les deux hommes monter vers lui. L'un était à cheval, l'autre à pied. (Camus: L'hôte, S. 105)

(8) On était au plein de l'hiver et cependant une journée radieuse se levait sur la ville déjà active. (Camus: Les muets, S. 79)

Diese und vergleichbare Fälle zeigen, dass das imparfait keine kontextuell gestützte Ankersituation benötigt. Vielmehr ist es das imparfait, das kraft seiner meronymisch-imperfektiven Semantik eine Ankersituation enthält, die es in (7) und (8) in den Textanfang einbringt. Der Kontext kann diese Situation näher präzisieren (etwa durch temporale Angaben), er konstituiert die Ankersituation aber nicht dies leistet der imperfektive Aspekt. Daher kann das imparfait seinen meronymi-

déjà saillante ou donnée comme déjà disponible“ (1993: 56; vgl. auch Berthonneau / Kleiber 1999: 154). Die Meronymie ist zugleich eine anaphorische Relation, bei der Sachverhalt und Situation über eine ,,anaphore associative“ (ebd.) verbunden sind.

19 Zur Frage, ob die anaphorisch-meronymische Semantik den imperfektiven Aspekt erzeugt oder umgekehrt vgl. Kleiber (2003; bes. 8, 10-12, 16 f.). Zur Gegenüberstellung von „approche anaphorique“ und ,approche aspectuelle“ vgl. auch Labeau (2002: 168-175) und Bres (2007: 23 f., 26 f.). 
schen Wert auch am Textbeginn entfalten, an dem kontextuell noch keine Ankersituation etabliert ist.

Die so skizzierte Bedeutung des imparfait und seine textsemantischen Profile werden in den folgenden beiden Abschnitten in zweifacher Weise kontrastiert: zum einen anhand der (in der Forschung omnipräsenten) Opposition von imperfektivem und perfektivem Aspekt, zum anderen im Vergleich zum présent.

\subsection{Imperfektiver und perfektiver Aspekt: imparfait und passé simple}

Die Bedeutung des imparfait konturiert sich besonders prägnant in der Aspektopposition zum passé simple, das eine perfektive Aspektbedeutung hat ${ }^{20}$. In Opposition zum imparfait versprachlicht der perfektive Aspekt Sachverhalte als zeitlich begrenzte Größen, die in der Vergangenheit zum Abschluss gekommen sind ${ }^{21}$. Diese perfektive Modellierung verdeutlichen die folgenden Beispiele:

(9) Par une entrée à droite de l'église, on peut aller visiter les pièces où vécut et mourut saint Ignace. (Guide Michelin: Rome, S. 110)

(10) Presque tout de suite après, au-dessus du vacarme de l'atelier, une sonnerie retentit deux fois. (Camus: Les muets, S. 92)

Das Charakteristikum der Begrenzung wird in Beispiel (9) deutlich, in dem auf Leben und Tod des Heiligen als sukzessive, in der Vergangenheit vollendete und abgeschlossene Ereignisse zurückgeblickt wird. Diese begrenzende Wirkung des perfektiven Aspekts manifestiert sich im Fall iterativer Sachverhalte (10) in der Begrenzung der Wiederholung (,deux fois“). Die aspektuelle Modellierung schlägt sich - analog zum imparfait - auch beim passé simple in einer kontinuierlichen und einer iterativen diskontinuierlichen Variante nieder. In der Kontrastierung von perfektivem und imperfektivem Aspekt wird so deutlich, dass das passé simple Sachverhalte versprachlicht, die dem Zeitfluss unterliegen und unter dem Gesichtspunkt der zeitlichen Veränderung als holistisch-begrenzte Ereignisse und Handlungen gesehen werden. Diese begrenzende Leistung des passé simple wird im nächsten Beispiel besonders deutlich, in dem es um ein römisches Adelsgeschlecht, die Farnese, geht:

20 Zur Opposition von imparfait und perfektivem passé simple vgl. etwa Confais (1990: 152-162) und Vetters (1996: 112-125, 142-152). Zugleich steht das imparfait auch in Opposition zum passé composé, vor allem wenn diese Form (in der Mündlichkeit und in bestimmten Traditionen des Schreibens) als perfektives Substitut für das passé simple verwendet wird. Zu imparfait vs. passé simple / passé composé im Französischen vgl. zusammenfassend Mitko (2000: 96-102). Da der perfektive Aspekt hier als verdeutlichendes Kontrastmittel zum imparfait dient, beschränke ich mich im Folgenden vereinfachend auf das passé simple. Zum passé composé (kontrastiv zum passé simple) vgl. Becker (2010b: bes. 4-6).

${ }^{21}$ Die zeitliche Begrenzung kann dabei eine Anfangs- und / oder Endterminierung sein. Zum perfektiven Aspekt im Französischen und zur Opposition von perfektivem und imperfektivem Aspekt vgl. etwa Imbs (1960: 84 f., 96 f.), Togeby (1982: 31-319) und Mitko (2000: 83-92). 
(11) Instigateur du concile de Trente, il fut le premier pape de la Contre-Réforme [...]. Lui-même et ses descendants réunirent une magnifique collection d'œuvres d'art. [...]. Le dernier des Farnese fut une femme, Elisabeth (1692-1766). (Guide Michelin: Rome, S. 113)

Der Textausschnitt gibt einen geschichtlichen Aufriss, in dem das passé simple die diskursive Organisation einer Sukzession leistet ${ }^{22}$. Der perfektive Aspekt präsentiert die Sachverhalte als Episoden einer Familiengeschichte. Dies erklärt auch die auf den ersten Blick ungewöhnlich erscheinende Wendung „Le dernier des Farnese fut une femme“. Das Faktum, dass der letzte Spross der Farnese eine Frau ist, wird hier nicht imperfektiv als „unbegrenzt seiende“ Eigenschaft einer Person präsentiert, sondern erscheint über die gezielte Selegierung des perfektiven Aspekts, als eine Etappe in der Genealogie der Familie. Es ist der perfektive Aspekt des passé simple, der hier „Geschichte macht“.

Besonders prägnant zeigt sich die Aspektopposition zudem in Beispielen, in denen für verwandte Sachverhalte in ähnlichen Kontexten aspektuell konträre Modellierungen gewählt werden. Dazu zwei korrespondierende Beispiele aus einer Literaturgeschichte:

(12) André Breton (né à Tinchebray, dans l'Orne, en 1896) - Breton se destinait d'abord à la médecine (pendant la Grande Guerre, il fut affecté à un service psychiatrique). Il entretint des relations personnelles avec le fameux Sigmund Freud [...]. (Merlot: Histoire de la littérature française, S. 185)

(13) Louis Aragon (né à Paris, en 1897) - Aragon se destina d'abord à la médecine. Puis il fut l'un des plus fougueux champions du surréalisme et déchira à belles dents toutes les valeurs „bourgeoises“. (Merlot: Histoire de la littérature française, S. 188)

Beide Textausschnitte entstammen Kurzbiographien und beinhalten den gleichen Sachverhalt (,se destiner à la médecine“), allerdings mit unterschiedlicher Darstellungsabsicht. In (12) wird die Entscheidung für das Medizinstudium als imperfektiv-unbegrenzte Disposition des Protagonisten versprachlicht, die den Rahmen für die folgenden sukzessiven Ereignisse setzt. In (13) dagegen ist „il se destina“ bereits die erste Station in der Lebensgeschichte Aragons und damit integraler Bestandteil einer in der Vergangenheit abgeschlossenen Sukzession.

Kontrastiv zeigen die beiden Textausschnitte, wie perfektiver und imperfektiver Aspekt unterschiedliche „Zeitgeschichten“ formen. Das passé simple ordnet Sachverhalte begrenzend in einen Zeitfluss ein und etabliert über die jeweiligen Begrenzungen eine in der Vergangenheit abgeschlossene Sukzession. Das imparfait dagegen blendet Begrenzungen aus, präsentiert Sachverhalte als unbegrenzt präsentisch zu einer Ankersituation und kann durch diese Präsentischkeit keine Sukzes-

22 Zum passé simple und seiner Fähigkeit, Sukzessionen zu etablieren vgl. Togeby (1982: 319) sowie de Saussure (2003: 237-246). Becker (2010b: 19-21) zeigt kontrastiv zum passé composé auf, dass die zentrale Funktion des passé simple die narratio als Wiedergabe sukzessiver Sachverhalte ist. 
sion generieren. Auf diese Weise hebt das imparfait Ereignisse und Handlungen aus dem Zeitfluss heraus. Es etabliert keine Sukzession, sondern erzeugt vielmehr eine Juxtaposition von Handlungen und Ereignissen. Während eine passé simpleSequenz eine zeitlich lineare Abfolge durchlaufener Sachverhalte in einem Zeitfluss darstellt, leistet das imparfait in Sequenz eine Überblendung von einem Sachverhalt zum nächsten, ohne Begrenzungen in den Blick zu nehmen. Aus dieser Charakterisierung folgt, dass das passé simple der weit bessere „Geschichtenerzähler" ist. Um zu verstehen, welche Wirkung das imparfait in narrativen Kontexten entfaltet, ist eine zweite Analogie entscheidend: die Parallelsetzung von imparfait und présent.

\section{Die Analogie von présent und imparfait}

Auf Parallelen zwischen imparfait und présent haben verschiedene Richtungen der Tempusforschung mehrfach aufmerksam gemacht. Die Parallelsetzung beider Formen wurde dabei primär als ordnendes Muster innerhalb des romanischen Verbalsystems verstanden ${ }^{23}$. Die Analogie kann jedoch noch in anderer Weise nutzbar gemacht werden, und zwar für eine semantisch-pragmatische Präzisierung des imparfait und seiner Funktionen ${ }^{24}$. Denn présent und imparfait haben gemeinsam, dass sie eine Ankersituation beinhalten und diese Situation in einen Text einbringen können, ohne weitere kontextuelle Stützungen zu benötigen. Im Falle des présent ist diese Ankersituation das ego-hic-nunc als deiktische origo, das imparfait dagegen beinhaltet eine zur origo vorzeitige Situation in der Vergangenheit ${ }^{25}$. Damit entspricht dem nunc des présent im Fall des imparfait ein in der Vergangenheit situiertes tunc.

Die aufgezeigte Analogie zum présent öffnet die Augen für eine wichtige Besonderheit der von beiden Formen mitkodierten Ankersituationen. So hat das présent durch seine Bezogenheit auf das mehrdimensionale ego-hic-nunc eine über das Zeitliche hinausgehende Aktualität, da die origo durch das Sprecher-ego immer auch eine personale Dimension enthält. Diese Koppelung von zeitlicher und personaler Dimension charakterisiert nun nicht allein das présent, sondern

23 Damourette / Pichon (1936: 162, 172-175, 207, 219-221) etablieren zwei um nunc und tunc gruppierte Zeitachsen, wobei dem présent das imparfait als „présent dans le passé“ entspricht. Coseriu (1976: 92-94) seinerseits strukturiert das romanische Verbalsystem in zwei Zeitebenen, wobei das aktuelle présent seinen Antipoden im inaktuellen imparfait hat. Vet (1980: 31-33; 2005: 33-41) schreibt dem Französischen ebenfalls ein binär strukturiertes Verbalsystem mit zwei Achsen zu, die um présent und imparfait zentriert sind.

24 Zur Semantik des présent, das durch den Bezug auf die Aktualität des Sprechens eine retrospektive und prospektive Perspektive auf sich vereint, vgl. Coseriu (1976: 93 f.), Togeby (1982: 311 f.) und Mellet (2001: 30-33). Zur Diskussion um die aspektuelle Markiertheit des présent vgl. Togeby (1982: 311) sowie Mellet (2000: 103 f., 2001: 32-35).

25 Zum Konzept des ego-hic-nunc und seinen Dimensionen vgl. Bühler ([1934] 1982: bes. 102, 107 f.). 
ebenfalls das imparfait ${ }^{26}$. Dabei entspricht dem Sprecher-ego in der personalen Dimension des présent auf Seiten des imparfait eine personale Größe, welche die sich im tunc vollziehenden Sachverhalte wahrnimmt und auf diese Weise die Position eines „Wahrnehmers“ einnimmt. Damit steht dem im nunc situierten Sprecher-ego in der vergangenen Situation ein wahrnehmendes Subjekt gegenüber, und der im nunc vollzogene Akt des Sprechens hat seine Entsprechung in einem in der Vergangenheit lokalisierten Akt der Wahrnehmung. Beiden Formen ist damit gemeinsam, dass sie mehrdimensionale Ankersituationen mitkodieren: Das présent evoziert das Zentrum des Sprechaktes, das imparfait dagegen das Zentrum eines Wahrnehmungsaktes. Diese Deutung des imparfait setzt damit analog zum présent bei der mehrdimensionalen Natur der Ankersituation an und ist in diesem Sinn genuin kommunikativ-pragmatisch. Die Analogie beider Formen und ihrer Ankersituationen verdeutlicht, dass die personale Dimension des imparfait weder ein kontextgebundener Effekt noch ein Epiphänomen ist ${ }^{27}$. Vielmehr ist die Verbindung von zeitlicher und personaler Dimension für die Semantik des imparfait ebenso zentral wie für die person- und zeitgebundene Aktualität des présent. Das Konzept der Ankersituation als in die Vergangenheit verschobenes „Ebenbild“ des ego-hic-nunc besitzt daher ebenfalls eine genuin mehrdimensionale Natur. Diese sich aus sprachpragmatischer Sicht erschließende zeitliche und personale Dimension des imparfait ist die Basis für ein imparfait, das ins Erzählen kommt.

\section{Wenn das imparfait ins Erzählen kommt ...}

\subsection{Vorüberlegungen}

Mit dem Begriff des imparfait narratif werden in der Forschung Verwendungen verbunden, in denen das imparfait in Kontexten auftritt, in denen eine perfektiv markierte Form - das passé simple (oder ggf. auch das passé composé) - erwartbar ist ${ }^{28}$. Dieser Kontextbindung ist die hier gewählte Formulierung vom imparfait in narra-

${ }^{26}$ Damourette / Pichon (1936: 177) definieren den „sens toncal“ als „le placement du fait verbal dans une autre sphère d'action, une autre actualité" und gehen damit ebenfalls über die rein zeitliche Dimension hinaus. Bei Vet (1980: 31-33; 2005: 33-41) entspricht dem „moment de l'énonciation“ der aktuellen Achse ein „point de perspective“ auf der inaktuellen Achse.

27 In der Forschung finden sich mehrfach Hinweise, dass das imparfait Sachverhalte in Anbindung an ein Subjekt versprachlicht, doch erscheint diese Anbindung als kontextbedingter Effekt der imparfait-Bedeutung: vgl. Pollak (21988: 139-141, 197), de Saussure / Sthioul (1999: 176, 184, 186; 2005: 108) und Tasmowski-de Ryck (1985: 73). Während Tasmowski-de Ryck von einem zeitreferentiellen Modell in der Tradition Reichenbachs ausgeht, inferieren de Saussure / Sthioul (1999: 184, 186) ein „sujet de conscience“ über logische und mengentheoretische Relationen.

${ }^{28}$ Für diese Verwendung finden sich ferner bspw. die Etiketten imparfait pittoresque, imparfait de rupture oder imparfait dynamique. Zur Vielfalt der Termini vgl. etwa Bres (1999a: 4), Berthonneau (2000: 74 f.) und Labeau (2005: 83). Eine Übersicht über die verschiedenen Termini gibt Labeau (2005: 101 f.). 
tiven Kontexten geschuldet ${ }^{29}$. Unter einem narrativen Kontext wird dabei ein Text oder Textabschnitt verstanden, in dem mindestens zwei Sachverhalte als Sukzession ikonisch in ihrer chronologischen Abfolge wiedergegeben werden ${ }^{30}$. Die narrativ organisierten Sachverhalte sind in semantischer und textueller Kohärenz verbunden und bilden als Sukzession eine Einheit ${ }^{31}$. Ein narrativer Kontext liegt vor, wenn die in einem Text oder Textausschnitt versprachlichten Sachverhalte sinnvoll nur als zeitlich begrenzte, aufeinander folgende Teile einer Sukzession und damit als dem Zeitfluss unterworfene Ereignisse verstanden werden können. Diese diskursive Organisation der sukzessiven Narration ist die zentrale Funktion des perfektiven passé simple, das daher in einem narrativen (genauer: narrativ-sukzessiven) Kontext die erwartbare Form darstellt ${ }^{32}$. Die Entwicklungslinien des imparfait narratif können hier nur skizziert werden. Überwiegend wird das imparfait in narrativen Kontexten als eine zu Beginn des 19. Jahrhunderts auftretende Innovation eingeordnet, die sich in den folgenden Jahrzehnten zunehmend etabliert ${ }^{33}$. Im Französischen der Gegenwart findet sich die Verwendung in den verschiedensten Textgattungen: im Roman, in historiographischen Texten, in Reiseführern, aber auch in Sportreportagen, Musikkritiken und anderen journalistischen Textsorten $^{34}$.

Die zentrale Frage ist, ob das imparfait in diesen Verwendungen tatsächlich wie eine perfektive Form funktioniert und damit die mit dem perfektiven Aspekt verbundene narrative Funktion erfüllt, oder ob es vielmehr bei seiner Verwendung in narrativ-sukzessiven Kontexten als imperfektiver Aspekt eine ganz eigene Wirkung entfaltet. Im Fall des imparfait in narrativen Kontexten werden im Folgenden zwei Typen unterschieden, die in verschiedener Weise kontextbedingt generiert

29 Diese Formulierung hat den Vorteil, dass sie offen lässt, ob hier eine vom gewohnten Profil abweichende Funktion des imparfait vorliegt oder ob es sich um einen kontextgebundenen Wert handelt.

30 Vgl. hierzu Stempel (1973: 326, 327 f., 329 f.) und Becker (2010a: 10, 19-21).

31 Nach Stempel (1973: 328, 329 f.) umfasst das narrative Minimalschema zwei aufeinanderfolgende Teilereignisse, die zusammen ein historisches Ereignis bilden, oder zwei in semantisch-pragmatischer Kohärenz stehende sukzessive (historische) Ereignisse.

32 Die umfangreiche Forschung zum imparfait narratif resümierende Darstellungen finden sich bei Imbs (1960: 92 f.), Togeby (1982: 341-345), Pollak (21988: 124-144, bes. 138), Vetters (1996: 126-128), Berthonneau (2000: 73, 77) und Bres (2005a: bes. 63-66, 2005b: 7-11, 15).

33 Zur Entstehung und Verbreitung vgl. Muller (1966: 256), Blumenthal (1986: 49-51, 100 f.) und Caudal / Vetters (2005: 47-49). Zur Diskussion um das Alter des imparfait narratif vgl. Muller (1966: 261), Blumenthal (1986: 49-51, 100-102), Weinrich ('2001: 135-140) und Hunnius (2009: 59). Analoge Verwendungen in narrativen Kontexten finden sich im Spanischen und Italienischen: vgl. Weinrich ('2001: 140 ff., 144 ff.), Bertinetto (1980: bes. 83, 88 f.) und García Fernández (2004: 72-75).

${ }_{34} \mathrm{Zu}$ den Textgattungen, die die Verwendung inaugurieren und verbreiten vgl. Muller (1966: 262), Togeby (1982: 341 f.), Blumenthal (1986: 101) und Pollak (21988: 125-127). 
werden und die sich auch hinsichtlich ihrer Wirkung unterscheiden: ein in der zeitlichen Dimension des tunc über Kollision und Kontrast operierender Typ und ein zweiter Typ, der bei der personalen Dimension der Ankersituation ansetzt.

\subsection{Kollision und Kontrast}

Der erste Typ, der über den Effekt von Kollision und Kontrast operiert, entspricht der Verwendung, die in der Forschung klassischerweise als imparfait narratif geführt wird ${ }^{35}$. Bei diesem Verwendungstyp erzeugt der imperfektive Aspekt des imparfait mit seiner Semantik der Unbegrenztheit im Kontext einer zeitliche Begrenzungen evozierenden narrativen Sukzession einen Effekt des Kontrasts bzw. einer Kollision zeitlicher Modellierungen ${ }^{36}$. Die folgenden Beispiele illustrieren in exemplarischer Weise diese Kollision von imperfektiver Unbegrenztheit und narrativ-kontextueller Begrenztheit:

(14) Dans la gare de Vizzavona [...], le célèbre bandit [...], à l'âge de 75 ans, se rendit en grande cérémonie à la justice; 20 ans plus tard il mourait chez lui, dans un hameau de Bocognano. (Guide Michelin: Corse, S. 140)

(15) En octobre 1765, l'écrivain écossais James Boswel arrivait à Sollacaro à la recherche de Paoli. Il fut durant plusieurs jours l'hôte du général [...]. Trois ans plus tard il publiait un essai sur „L'État de la Corse“ [...]. (Guide Michelin: Corse, S. 130)

Beide Textausschnitte haben gemeinsam, dass im imparfait Ereignisse und Handlungen versprachlicht werden, die Bestandteile zeitlicher Sukzessionen sind. Erzählt werden herausgehobene Stationen im Leben eines korsischen Banditen (14) bzw. Episoden aus dem Leben eines Schriftstellers (15). Diese zeitliche Sukzession wird im Text durch verschiedene sprachliche Mittel etabliert. In beiden Textausschnitten setzen temporale Angaben zeitliche Begrenzungen (,20 ans plus tard“, „trois ans plus tard“) und etablieren so eine narrative Sukzession ${ }^{37}$. Der Charakter einer Sukzession wird auch durch die Verbsemantik bzw. die im Text versprachlichten Situationstypen verstärkt. Die hier verwendeten telischen Verben (mourir, arriver, publier) sind grundsätzlich mit dem perfektiven und dem imperfektiven Aspekt kompatibel, doch hat die telische Semantik der Zieleinlösung, die das Konzept einer Grenze impliziert, eine Affinität zum (perfektiven) Ausdruck von Sukzessionen ${ }^{38}$. Daher überrascht es nicht, dass sich beim imparfait narratif

35 Vgl. etwa Togeby (1982: 341), Pollak (21988: 125), Berthonneau (2000: 74 f.) und Labeau (2005: 83, 101 f.).

36 Zum Konzept der Kollision imperfektiver Aspekt vs. Kontext der Abgeschlossenheit vgl. Togeby (1982: 341), Blumenthal (1986: 102 f.), Labeau (2005: 96 f.) und Bres (2005a: 9).

$37 \mathrm{Zu}$ Funktion und Rolle temporaler Angaben beim imparfait narratif vgl. Togeby (1982: 342 f.), Tasmowski-de Ryck (1985: 63, 67), Berthonneau / Kleiber (1999: 152 f.), Berthonneau (2000: 96-98, 100-105) und Bres (2005a: 90-96, 105-127, 2005b: 9-14).

$38 \mathrm{Zu}$ dieser Affinität vgl. Mitko (2000: bes. 196-199). 
eine ausgeprägte Affinität zu telischen Verben bzw. zu Sachverhalten findet, die als Situationstyp eine (grenzsetzende) Zieleinlösung enthalten ${ }^{39}$.

Berücksichtigt man die eingangs getroffene Unterscheidung zwischen punktuellen und flächigen Interaktionen von Aspektform und Kontext, dann liegt im Fall der temporalen Angaben und der telischen Verben eine punktuelle Form des Zusammenspiels vor. Zugleich verdeutlichen die beiden Textausschnitte, dass der narrative Kontext ebenfalls durch die Textgattung vorgegeben wird. Die Beispiele (14) und (15) entstammen Kurzbiographien, in denen in chronologischer Reihenfolge die zentralen Stationen eines Lebens genannt werden. Diese den Text prägende Diskurstradition der narratio stellt ein flächiges Phänomen dar, da die Textgestalt hierdurch in ihrer Gesamtheit charakterisiert wird. Damit sind beide Textausschnitte sowohl punktuell durch temporale Angaben als auch flächig durch die Gattung und ihre Diskurstradition(en) als narrative Kontexte markiert.

Der imperfektiven Aspektsemantik des imparfait steht damit ein ganzes Bündel begrenzender Parameter gegenüber. Die ausgeprägte Markierung als Narration, die einen perfektiven Aspekt erwarten lässt, kontrastiert in markanter Weise mit dem imparfait. Während der Kontext festlegt, dass das Ziel der (telischen) Handlungen erreicht wurde, blendet das imparfait diese Zieleinlösung aus. Auf diese Weise wird einem Kontext, der eine abgeschlossene Zieleinlösung impliziert, ein imperfektiver Aspekt entgegengesetzt, der diese Zieleinlösung in einem Akt der Unterspezifizierung offen lässt. Dieser Kontrast ist es, der in der zeitlichen Dimension der Ankersituation den Effekt einer Kollision erzeugt. Die Kollision ist damit kein bloßer Überraschungseffekt einer ,unerwarteten“ Form. Entscheidend ist die Kombination eines zeitliche Grenzen setzenden Kontextes mit dem keine Begrenzungen spezifizierenden imparfait ${ }^{40}$. Darüber hinaus leisten die für das imparfait narratif charakteristischen temporalen Angaben durch die explizite Benennung der Ankersituation eine Betonung der zeitlichen Dimension. Diese Fokussierung der Zeitlichkeit lässt nun die Kollision zwischen begrenzendem Kontext und entgrenzendem imparfait noch deutlicher hervortreten und verschärft so den Kontrast zwischen einem imparfait der Juxtaposition und einem Kontext der Sukzession. Die Grundlage für diesen Gebrauch ist die zeitliche Dimension der im imparfait enthaltenen Ankersituation, die durch Kontextfaktoren in den Vordergrund gerückt wird. Im Unterschied dazu basiert der zweite Verwendungstyp auf der personalen Dimension der vom imparfait präsupponierten Ankersituation.

39 Diese Affinität zu telischen Verben konstatieren u. a. Muller (1966: 267), Pollak (21988: 2-30), Berthonneau / Kleiber (1999: 144), Berthonneau (2000: 80 f., 92-95) und Bres (2005a: 88-90).

40 Dieser Kollisionseffekt wird überwiegend anhand schriftlicher Texte dokumentiert, er funktioniert jedoch auch in mündlichen Erzählungen: „A 9 heures 10, Noëlle est partie. 10 minutes plus tard Jean-Luc arrivait“ (Hausmann 1997: 177). 


\subsection{Personale Dimension und Wahrnehmung}

Grundlage für den zweiten Verwendungstyp ist, dass das imparfait eine vergangene Situation als Ankersituation präsupponiert, die neben der zeitlichen auch eine personale Dimension einschließt und daher mit einem Akt der Wahrnehmung und der personalen Größe eines Wahrnehmers verbunden ist. Die Eigenschaft des imparfait, durch seine personale Dimension einen Wahrnehmer ins Spiel zu bringen, illustriert das folgende Beispiel:

(16) Mais avant qu'il [Yvars] les eût rejoints, ils se retournèrent soudain vers les portes de l'atelier qui venaient de s'entrouvrir. Ballester, le contremaître, apparaissait dans l'embrasure. Il ouvrait l'une des lourdes portes et, tournant alors le dos aux ouvriers, la poussait lentement sur son rail de fonte. (Camus: Les muets, S. 87)

Der Text schildert die Ankunft des Protagonisten Yvars bei den wartenden Arbeitern. Das Handlungsgerüst beinhaltet eine narrative Sukzession: Yvars kommt an, die wartenden Arbeiter drehen sich zur Türe der Werkstatt, der Vorarbeiter Ballester erscheint im Türrahmen, öffnet einen Türflügel und schiebt ihn auf. Da diese Sequenz allein als dem Zeitfluss unterworfene narrative Sukzession gedeutet werden kann, treten die imparfait-Formen hier in einem narrativen Kontext auf. Der Kontext etabliert einen markanten Zeitfluss, das verwendete imparfait jedoch hebt die einzelnen Handlungen durch seine meronymisch-imperfektive Semantik, welche die Sachverhalte als „unbegrenzt präsentisch seiend“ zu einer Ankersituation versprachlicht, aus dem kontextuell gegebenen Zeitfluss heraus. Auch in diesem Fall beinhaltet der Textausschnitt eine zeitliche Kollision zwischen dem imperfektiven imparfait und der einen perfektiven Aspekt fordernden narratio, doch trifft die auf die zeitliche Dimension rekurrierende Erklärung nicht den Kern des hier vorliegenden Effekts. Vielmehr gründet die Wirkung in der personalen Dimension des imparfait, die einen Akt der Wahrnehmung und damit die Perspektive eines Wahrnehmers enthält. Durch diese personale Dimension präsentiert das imparfait die Aktionen Ballesters als Handlungen, die Gegenstand eines Wahrnehmungsaktes sind. Die im imparfait versprachlichten Sachverhalte sind durch die präsupponierte Ankersituation zugleich immer auch präsentisch zu einem Wahrnehmungsakt. Im zitierten Textausschnitt wird dieser Wahrnehmungsakt kontextuell gestützt, da der Text die „Wahrnehmer“ explizit benennt: Es sind die Arbeiter als Protagonisten der Erzählung, die beobachten, wie Ballester im Türrahmen erscheint, den Türflügel allmählich öffnet und aufschiebt. Der Wahrnehmungsakt bzw. Sehakt kann also über die Nennung der die Handlungen beobachtenden Arbeiter inferiert werden ${ }^{41}$. Dabei wird jede einzelne Handlung als eine für

41 Zur Erklärung können auch Strukturen des Typs „Il aperçut Janvier qui sortait d'une boutique de légumier" herangezogen werden, die das imparfait verlangen, da das Wahrgenommene immer präsentisch zum syntaktisch übergeordneten Akt des Wahrnehmens ist (Mitko 2000: 131, vgl. auch Togeby 1982: 321). Diese Struktur wird beim imparfait 
sich wahrgenommene Aktion präsentiert und damit an einen Wahrnehmungsakt gebunden. Diese Rückbindung an ein wahrnehmendes Subjekt bewirkt, dass die von Ballester realisierten Aktionen auf der Ebene der Textsemantik nicht als eine dem Zeitfluss unterworfene Sukzession erscheinen, sondern vielmehr als vereinzelte, in Juxtaposition stehende Handlungen, wobei jeder Handlung ein eigener Wahrnehmungsakt zugrunde liegt. Auf diese Weise löst das imparfait Sachverhalte, die textsemantisch allein als Sukzession verstanden werden können, aus ihrer narrativ-sukzessiven Ordnung. Die Sachverhalte erscheinen - über die zeitliche Kontrastierung von kontextueller Begrenzung und entgrenzendem imparfait hinaus - als von einem Subjekt wahrgenommene und in diesem Sinne perspektivierte Sachverhalte. Auch das folgende Beispiel illustriert eine Verwendung in einem narrativen Kontext, in dem das imparfait eine an ein wahrnehmendes Subjekt gekoppelte Perspektivierung leistet:

(17) Rieux avait écouté le journaliste avec attention. Sans cesser de le regarder, il dit avec douceur: - „L'homme n'est pas une idée, Rambert“. L'autre sautait de son lit, le visage enflammé de passion. (Camus: La peste, S. 151)

Die im Textausschnitt geschilderte Handlung Ramberts ist eine Reaktion auf die Worte Rieuxs, die erst erfolgt, nachdem dieser zu Ende gesprochen hat. Das Aufspringen Ramberts ist damit eine neu einsetzende, in der Zeit begrenzte Handlung. Diese Aktionen können allein als Sukzession in der Zeit interpretiert werden, sodass ein narrativer Kontext gegeben ist, der einen perfektiven Aspekt erwarten lässt - eine Erwartung, die durch den imperfektiven Aspekt konterkariert wird ${ }^{42}$. In analoger Weise wie in Beispiel (16) dient auch hier das imparfait dazu, die Handlung an einen Wahrnehmungsakt und damit an die Perspektive eines wahrnehmenden Subjekts anzubinden. Auch in diesem Textausschnitt wird der Sehakt nicht explizit genannt, kann jedoch inferiert werden. So wird Rieux als ein Gesprächspartner beschrieben, der sein Gegenüber genau beobachtet („sans cesser de le regarder, il dit [...]“). Über diese Thematisierung des Beobachtens wird der für die imparfait-Verwendung entscheidende Akt des Wahrnehmens konstituiert: Rieux wird als wahrnehmendes Subjekt eingeführt; er ist es, dessen Augen sich das Bild des impulsiv aufspringenden Rambert bietet.

Beiden Textausschnitten (16) und (17) ist gemeinsam, dass agierende Protagonisten als beobachtende, wahrnehmende Subjekte in einer Weise inszeniert werden, dass der Wahrnehmungsakt im Text in „Spurenelementen“ angelegt und entsprechend inferierbar ist. Diese Spurenelemente eines Wahrnehmungsaktes

narratif gleichsam erweitert: Die Verwendung löst sich von der (syntaktischen) Korrelation an einen Wahrnehmungsakt und kann stattdessen ohne explizite syntaktische Anbindung auf im Text präsente Sinneseindrücke eines Subjekts bezogen werden.

42 Dieser Textausschnitt ähnelt Beispiel (6), allerdings mit einem wichtigen Unterschied. In (6) ist die Geste der Mutter („Ma mère haussait doucement les épaules“) redebegleitend und damit präsentisch zur Replik des Vaters, sie ist keine Reaktion auf dessen Worte, sodass in (6) kein narrativer Kontext und damit auch kein imparfait narratif vorliegt. 
können nun noch subtiler angelegt sein, wie der folgende Ausschnitt aus einem Simenon-Roman belegt:

(18) Et le miracle se produisait enfin, le plus naturellement du monde, si naturellement que c'en était déroutant. La porte du café s'ouvrait et se refermait aussitôt tandis que la Marie se profilait sur le seuil. Elle y restait un moment, le temps de mettre son manteau sur sa tête, comme font les filles du pays quand il pleut. (Simenon: La Marie du port, S. 66 f.)

Der Textausschnitt schildert nach dem rhetorischen Muster von Summe und Detail das Erscheinen der Protagonistin Marie als wunderbare Begebenheit: Die Tür öffnet und schließt sich, Marie erscheint auf der Schwelle und bleibt dort kurz stehen. Die versprachlichten Handlungen können damit allein als in einer Relation der zeitlichen Sukzession stehende Aktionen begriffen werden. Auch die einleitend-summierende Bewertung der Sequenz als miracle gilt einem einmaligen Ereignis und fördert so die Semantik der Abgeschlossenheit in der Vergangenheit. Damit ist in diesem Text ebenfalls ein narrativer Kontext gegeben, der mit dem imperfektiven Aspekt kollidiert. Doch auch hier beschränkt sich der Effekt nicht auf einen zeitlichen Kontrast, sondern gründet wie in (16) und (17) in der personalen Dimension und dem von ihr transportierten Wahrnehmungsakt. Vor der Folie der bereits interpretierten Beispiele stellt sich die Frage, in welcher Weise im Simenon-Text der Sehakt erschließbar ist, denn anders als in den bisher besprochenen Fällen findet sich im unmittelbaren Kontext der imparfait-Verwendung keine (den beiden Camus-Texten vergleichbare) lokale Inszenierung eines Wahrnehmungsaktes. Im Fall des Simenon-Romans sind Sehakt und wahrnehmendes Subjekt jedoch ebenfalls gegeben, allerdings treten sie in der Gestaltung der fiktionalen Situation in indirekterer Weise als in (16) und (17) in Erscheinung. Die Rolle des Beobachters ist im Simenon-Roman ebenfalls durch eine fiktionale Figur gefüllt (der Protagonist Marcel hofft auf ein Treffen mit Marie und beobachtet sie), doch ist der Wahrnehmungsakt nicht über eine Thematisierung des Sehens, sondern lediglich über die den zitierten Textausschnitt gleichsam einrahmende Wiedergabe der Gedanken des Protagonisten erschließbar: Es sind vor allem die (überwiegend im discours indirect libre wiedergegebenen) Überlegungen des Protagonisten, die dessen Präsenz vermitteln. Die den Textausschnitt prägende Technik des Erzählens, die für diesen und andere Romane aus der Feder Simenons charakteristisch ist, basiert darauf, dass Sehakt und wahrnehmendes Subjekt zwar auch in der fiktionalen Situation der histoire gegeben sind, zugleich jedoch entscheidend durch die Erzählweise und damit durch die Ebene des discours konstruiert werden ${ }^{43}$. Wahrnehmendes Subjekt und Sehakt sind also nicht

43 In Simenon-Romanen findet sich häufig das Szenario, dass ein Protagonist Handlungen und Ereignisse beobachtet - nicht zufällig spielt im Werk Simenons die Figur des aufmerksamen Beobachters in der Gestalt des Protagonisten Maigret eine so herausgehobene Rolle. Zur Rolle des wahrnehmenden Beobachters bei Simenon vgl. auch Muller (1966: 265-267), Tasmowski-de Ryck (1985: 70) und Mitko (2000: 131 f.). 
allein durch die fiktionale Figur getragen, sondern werden auch durch die Technik der Perspektivierung erzeugt. Im Umkehrschluss ergibt sich, dass die Verwendung des imparfait großen Anteil an dieser Technik hat: Denn durch seine Kraft, in der personalen Dimension der Ankersituation ein wahrnehmendes Subjekt mitzukodieren, trägt das imparfait entscheidend dazu bei, dass die geschilderten Sachverhalte in einem Sehakt gespiegelt erscheinen. Damit ist das imparfait durch die Etablierung eines Wahrnehmers und die Anbindung an einen Sehakt diejenige Form, welche die perspektivierende Erzählweise maßgeblich ermöglicht und prägt.

Diese Wirkung des imparfait kann in einer kontrastiv-textvergleichenden Analyse weiter verdeutlicht werden, und zwar anhand zweier Artikel aus den Tageszeitungen Le Figaro und L'Humanité, in denen über das gleiche Ereignis - einen aufsehenerregenden Einbruch - berichtet wird ${ }^{44}$. Der große Vorteil einer solcherart vergleichenden Analyse liegt darin, dass beide Texte grosso modo der gleichen journalistischen Textgattung des Berichts angehören und zudem die gleiche Sukzession von Ereignissen abbilden. Durch diese Variation wird die Differenzqualität der Aspektwahl kontrastiv besonders deutlich:

(19) Après s'être emparé de son butin, le voleur sortit de sa poche une pelote de ficelle avec laquelle il ligota la jeune femme. Puis, l'ayant également bâillonnée, il l'allongea sur le lit des époux Reynolds avant de repartir par la terrasse. (Le Figaro)

(20) Après s'être emparé de son butin (deux mallettes dissimulées dans le boudoir $[\ldots])$, le voleur sortait de sa poche une pelote de ficelle, ligotait la malheureuse domestique, et repartait par la terrasse, en passant par une autre fenêtre. (L'Humanité)

Im Vergleich beider Textausschnitte springt ins Auge, dass im Figaro-Text das passé simple verwendet wird, wogegen bei weitgehend identischer Schilderung des Einbruchs in L'Humanité das imparfait gewählt wird ${ }^{45}$. Auch liegt beiden Versionen das gleiche Handlungsgerüst zugrunde: Der Einbrecher schnappt sich die Beute, holt ein Stück Schnur aus seiner Tasche, fesselt die Hausangestellte und flieht über die Terrasse. Beide Texte sind also durch die gleiche narrative Diskursorganisation charakterisiert und verbalisieren Handlungen, die nur als eine dem Zeitfluss unterworfene Sukzession deutbar sind.

44 Die beiden Berichte erschienen zeitgleich Ende der 1980er Jahre in den beiden Zeitungen. Den Hinweis auf dieses Variantenpaar verdanke ich Claus Morgenstern. Beide Texte sind im Ton der Reportage verfasst; zur Typologie journalistischer Textsorten vgl. Kött (2004: 13).

45 Vermutlich gehen beide Texte auf eine gemeinsame Quelle (die Meldung einer Presseagentur) zurück. Abgesehen von der abweichenden Aspektverwendung finden sich weitere (jenseits von Tempus und Aspekt) aufschlussreiche Divergenzen: So erwähnt Le Figaro die von den Einbrechern erbeuteten Reichtümer nur knapp, während der Bericht in L'Humanité geradezu in den aufgehäuften (kapitalistischen) Kostbarkeiten schwelgt. 
Grundsätzlich wirft die Existenz zweier Versionen, in denen eine identische Handlungsstruktur in einem Fall durch das perfektive passé simple, im anderen durch das imperfektive imparfait wiedergegeben wird, die Frage nach einer möglichen Abschleifung der Aspektopposition auf, und es gilt zu prüfen, ob hier möglicherweise eine „freie“ Variation vorliegt, die nicht (mehr) an verschiedene Textprofile - Sukzession vs. Juxtaposition - gekoppelt ist. Die abweichende Aspektwahl erklärt sich jedoch in diesem Fall durch die Wahl unterschiedlicher Techniken der Diskursorganisation. Beide Textpassagen erzeugen nämlich verschiedene Effekte. Das im Figaro gewählte (erwartbare) perfektive passé simple gibt die einzelnen Teilhandlungen des Einbruchs als sukzessive, in einem Zeitfluss stehende Aktionen wieder. Anders das imparfait: Es funktioniert auch in (20) als eine Aspektform, die kraft ihrer Imperfektivität und ihrer personalen Dimension ein wahrnehmendes Subjekt mitkodieren $\mathrm{kann}^{46}$. Auf diese Weise gibt das imparfait die Ereignisse perspektiviert als Handlungen wieder, die von einem Beobachter wahrgenommen wurden.

Wie die Beispielreihe zeigte, kann diese Beobachterrolle an den Wahrnehmungsakt eines fiktionalen Protagonisten gebunden sein, wie dies in den Textausschnitten (16) und (17) der Fall ist, in denen Spurenelemente eines Wahrnehmungsaktes gegeben sind - sei es die explizite Nennung der Wahrnehmer (16) oder des Sehaktes (17) im unmittelbaren Kontext des imparfait narratif. Der SimenonText (18) illustriert hier eine Dehnung dieses Verfahrens, denn die Anbindung an den Wahrnehmer erfolgt nur zum Teil über Referenzen auf den fiktionalen Protagonisten, vielmehr wird die Präsenz des wahrnehmenden Subjekts in entscheidender Weise durch die gewählte Technik des Erzählens konstituiert. Diese indirekte Anbindung an einen Beobachter wird im journalistischen Text (20) nun noch gesteigert, denn die im Text wiedergegebenen Ereignisse geschahen selbstverständlich nicht unter den Augen eines das Verbrechen observierenden Zeugen. Und doch ist es im Repertoire der journalistischen Textgattung gerade die Fähigkeit des imparfait, einen Wahrnehmer zu evozieren, die hier ausgespielt wird ${ }^{47}$. Denn die

46 Zwei nahezu identische Varianten zitiert auch Togeby (1982: 345): „Cotin n'eut pas la force de protester. Dix ans plus tard, il mourut dans l'obscurité." (René Bray: Molière, homme de théâtre, 1954, S. 232) vs. „L'abbé Cotin, écrasé, ne réplique pas; dix ans plus tard, il mourait dans l'obscurité." (René Bray: Molière, Euvres complètes VIII, 1952, S. 278 note de René Bray). Während in der ersten Variante sukzessiv erzählt wird, erzeugt das imparfait im zweiten Zitat im Zusammenspiel mit der temporalen Angabe einen Kollisionseffekt und damit eine andere Nuance der Textgestaltung, die dem zeitlichen Typ des imparfait narratif entspricht.

47 In journalistischen Textsorten dient das imparfait narratif häufig dazu, einen Beobachter zu konstruieren, dessen Rolle dann mit dem Kommentator, der über die Ereignisse berichtet, zusammenfällt. Zur Verwendung des imparfait in journalistischen Textsorten vgl. Muller (1966: 255), Tasmowski-de Ryck (1985: 65-67, 72 f.) und Berthonneau (2000: 82). 
Bindung an einen Sehakt suggeriert die Präsenz eines Beobachters und erzeugt auf diese Weise die Nuance eines Augenzeugenberichts - ganz so als sei ein Wahrnehmer als Zeuge vor Ort gewesen ${ }^{48}$. Damit fungiert die vom imperfektiven Aspekt geleistete Evokation eines Wahrnehmungsaktes zugleich als (journalistische) Beglaubigungsstrategie ${ }^{49}$. Diese Nuance ist bei der Le Figaro-Version im passé simple nicht gegeben - hier erscheinen die Teilhandlungen des Einbruchs durch den perfektiven Aspekt lediglich als eine holistisch-abgeschlossene Sukzession in der Vergangenheit ${ }^{50}$. Die unterschiedliche Aspektverwendung in beiden Textvarianten ist also durch unterschiedliche journalistische Erzähltraditionen bedingt.

\subsection{Zwei Typen des imparfait in narrativen Kontexten}

Bei der Verwendung des imparfait in narrativ-sukzessiven Kontexten lassen sich zwei Verwendungstypen unterscheiden. Der erste Typ rekurriert auf die zeitliche Dimension der vom imparfait mitkodierten Ankersituation und akzentuiert Kontrast und Kollision zwischen einem narrativen Kontext, der Grenzen setzt und einem imparfait, das durch seinen imperfektiven Aspekt eine entgrenzende Wirkung hat. Der zweite Typ dagegen nutzt die personale Dimension; er arbeitet mit dem Konzept des Wahrnehmers, leistet eine Anbindung an einen Sehakt und stellt als Technik der Erzählung den komplexeren Typ dar. Dieser Zusammenhang wird deutlich, wenn man die Parallele zum ego-hic-nunc konsequenter als bisher weiterdenkt und die personale Dimension ernst nimmt. Erst diese ganzheitliche Sicht erlaubt es, das Wirken des imparfait in narrativen Kontexten differenziert zu beschreiben.

48 Coseriu (1976: 140) weist darauf hin, dass das imparfait narratif im „,italienischen Verwaltungsstil, beispielsweise in Polizeiberichten“ verwendet werde (Beispiel: „Un ladro entrava nella casa ... La polizia si recava sùbito nella casa anzidetta e arrestava il malfattore ..."). Auch in diesem Fall wirkt die imperfektive Form als Beglaubigungsstrategie.

49 In der Forschung findet sich bei Tasmowski-de Ryck (1985: 73), die mit einer zeitreferentiellen Systematik argumentiert, sowie bei Berthonneau (2000: 84) der Verweis auf einen „moment de perception“, der in journalistischen Texten an einen Kommentator, in fiktionalen Texten dagegen an einen fiktionalen Protagonisten gebunden sei.

50 Die mögliche Hypothese, die Wahl des imparfait in L'Humanité sei eine Abwahl des (als konservativ erachteten) passé simple, erscheint aus zwei weiteren Gründen nicht plausibel. Zum einen hätte sich als Alternative eher das passé composé angeboten, zum anderen ist das passé simple im fraglichen Jahrzehnt (den 1980er Jahren) in der L'Humanité durchaus vital vertreten, wie korpuslinguistische Auswertungen zeigen. So analysiert Labeau (2007: 210) passé simple-Frequenzen vergleichend in Le Monde und L'Humanité und stellt fest, dass das passé simple in den 1980er Jahren in der L'Humanité deutlich häufiger verwendet wird als in Le Monde. Eine prinzipiell zu bedenkende sprachpolitisch motivierte Vermeidung des passé simple erscheint also unwahrscheinlich. 
Die zwei Typen unterscheiden sich darüber hinaus darin, dass sie die Ankersituation mit unterschiedlicher Explizitheit bzw. Implizitheit evozieren. So ist eine ausgeprägte Explizitheit der zeitlichen Situierung mit einer Betonung der zeitlichen Dimension gekoppelt. Dagegen geht eine hohe Implizitheit der Ankersituation mit einer Fokussierung der personalen Dimension einher. Setzt man hinsichtlich der Ankersituation ein Kontinuum zwischen Explizitheit und Implizitheit an, dann nutzen die vorgestellten Typen gleichsam die Pole dieses Kontinuums: Während der erste Verwendungstyp bei hochgradiger Explizitheit des in aller Regel durch eine temporale Angabe versprachlichten tunc ansetzt, nutzt der zweite Typ eine hochgradig implizite Ankersituation und fokussiert deren personale Dimension. Innerhalb des zweiten Typs unterscheiden sich die Verwendungen hinsichtlich der Art und Weise, wie Wahrnehmungsakt und Wahrnehmer im Text angelegt bzw. inferierbar sind. Wie die Beispielreihe zeigt, kann die imparfait-Verwendung an Textelemente auf récit-Ebene anknüpfen, die einen Wahrnehmungsakt beinhalten oder in mehr oder weniger komplexer Weise inferierbar machen. Die imparfait-Verwendung kann jedoch auch zu einem großen Teil auf einer Technik des Erzählens basieren, die eine perspektivierende Wahrnehmung beinhaltet. Betrachtet man die beiden hier differenzierten Typen des imparfait in narrativen Kontexten im Licht der Erzähltheorie, dann ist der erste Typ nicht an eine bestimmte Erzähltechnik gekoppelt; die Ereignisse und Handlungen werden hier nicht notwendig aus der Perspektive eines Wahrnehmers präsentiert. Der auf Kollision und Kontrast basierende Typ beinhaltet also keine erzähltheoretische Festlegung. Beim zweiten Typ dagegen ist aufgrund des evozierten Wahrnehmungsaktes eine Perspektivierung des Erzählens gegeben, deren Träger ein in der erzählten Situation präsenter Protagonist bzw. eine durch Erzähltechnik oder Textgattung konstituierte Sicht eines Wahrnehmers ist.

Für den Vergleich der beiden Verwendungstypen - zeitliche Dimension und Kollision vs. personale Dimension und Fokussierung des Wahrnehmungsaktes kann auch das eingangs erwähnte Begriffspaar der punktuellen vs. flächigen Interaktion zwischen Aspektform und Textumgebung herangezogen werden. So gründet die zeitliche Kollision zu einem großen Teil auf dem Kontrast zwischen den imparfait-Formen und den für diese Verwendung hochgradig frequenten temporalen Angaben, die eine zeitlich begrenzende Situierung leisten. Der Kontrast zwischen Aspektform und temporaler Angabe rührt damit von einzelnen, eingrenzbaren Formen im Text her und ist punktueller Natur. Daneben basiert die zeitliche Kollision in vielen Fällen zugleich auf einer narrativ-sukzessiven Diskursorganisation, die den Text oder Textabschnitt in seiner Gesamtheit prägt und daher flächig angelegt ist. Damit ist der erste Typ des imparfait in narrativ-sukzessiven Kontexten eine Verwendung, bei der die imparfait-Formen und damit der imperfektive Aspekt in punktueller und flächiger Weise mit dem Kontext interagieren. Anders der zweite Typ, bei dem die personale Dimension im Fokus steht. Bei diesem Typ ist die imparfait-Verwendung an eine Erzähltechnik gebunden bzw. der imperfektive Aspekt wird eingesetzt, um einen Sehakt zu konstituieren und so eine perspektivische Erzählweise zu erzeugen. Dieses Phänomen prägt als Technik der Textge- 
staltung den gesamten Text und ist daher durch eine flächige Wirkungsweise charakterisiert. Während der erste Typ auf der Ebene der Textsemantik also sowohl punktuell als auch flächig funktioniert, ist der zweite Typ primär flächig angelegt.

\section{Die Zeiten ändern sich: die Traditionalität des imparfait in narrativen Kontexten}

Die analysierten imparfait-Verwendungen in narrativen Kontexten illustrieren, dass sich das imparfait in diesen Fällen - kontrastiv zum passé simple - in den Dienst von Erzähltechniken stellt, die den narrativen Diskurs in anderer Weise organisieren als das passé simple, das eine sukzessiv-narrative narratio leistet. Während der erste Typ mit der Kollision von begrenzender Sukzession und entgrenzender Juxtaposition spielt, hat der zweite Typ großen Anteil an der Gestaltung einer an die Rolle eines Wahrnehmers gebundenen Erzählweise. $\mathrm{Zu}$ dieser perspektivierenden Erzählweise kann das imparfait aufgrund seiner meronymischimperfektiven Semantik und dank der personalen Dimension der ihm inhärenten Ankersituation beitragen. Ein wichtiger Parameter für die Aspektwahl ist also, welche Erzähltradition in einem Text und einer Textgattung gewählt wird. An diesen Befund schließt sich die Frage an, inwiefern sich „die Zeiten ändern“, wenn das imparfait in narrativen Kontexten zum Einsatz kommt. Die Qualität der hier dargelegten Veränderung wird deutlich, wenn man die Traditionalität des imparfait in den Blick nimmt und die Traditionen aufschlüsselt, in denen es steht. Als Aspektform gehört das imparfait zum temporal-aspektuellen System des Französischen und ist damit auf der einzelsprachlichen Ebene anzusiedeln. Die anhand der Beispiele diskutierten Techniken des Erzählens dagegen nutzen als Diskurstraditionen zwar sprachliche Mittel wie Aspektformen, stellen jedoch selbst kein sprachliches Wissen, sondern ein auf Sprache und Sprachverwendung in Texten bezogenes kulturelles Wissen dar. Die Technik, in der Vergangenheit abgeschlossene Ereignisse holistisch als in der Vergangenheit eingekapselte Sukzession zu versprachlichen und dazu das passé simple zu verwenden, ist eine kulturelle Diskurstradition, und auch die vom imparfait in narrativen Kontexten realisierte spannungsreiche Auflösung dieser Sukzession ist eine diskurstraditionelle Technik. Beide Wissensbestände - das einzelsprachliche und das diskurstraditionelle Wissen - unterliegen historischem Wandel. Wenn sich die Zeiten ändern, dann kann dies in zweierlei Weise geschehen: Die Veränderung kann das einzelsprachliche Wissen oder aber die kulturelle Traditionalität der Diskursorganisation betreffen. Die in zwei Typen gebündelten Effekte des imparfait in narrativen Kontexten operieren beide (kontrastierend) mit dem imperfektiven Aspekt und setzen daher eine intakte imperfektive Markierung voraus. Wenn das imparfait ,ins Erzählen kommt", dann ändert sich nicht die einzelsprachliche Bedeutung des imparfait, sondern es bilden sich vielmehr veränderte kulturelle Diskurstraditionen des Erzählens heraus, welche die imparfait-Bedeutung in neuer Weise nutzen. Als Ausdruck der Juxtaposition löst das imparfait in den Texten die Linearität in der Erzählung auf, was dann mit der Betonung des Sehakts und der Perspektive in 
den entsprechenden Textgattungen (etwa im Roman des Realismus) korrespon$\operatorname{diert}^{51}$. Die Veränderung liegt damit nicht im einzelsprachlichen Wissen, sondern in der Domäne der Diskurstraditionen als denjenigen Techniken, mit denen Tempus- und Aspektformen aus dem einzelsprachlichen System selegiert und in Texten arrangiert werden. Es sind die Diskurstraditionen des Erzählens, die sich wandeln und die imparfait-Bedeutung in neuer Weise „ausbeuten“, um in den Texten die lineare Ordnung der Sukzession durch eine Technik der Juxtaposition zu ersetzen. Das imparfait reüssiert daher in Gattungen und Erzähltraditionen, welche die Linearität des Erzählens auflösen, wie das seit dem 19. Jahrhundert der Fall ist. Die Mitkodierung des Wahrnehmungsaktes beim imparfait korrespondiert hier mit der Betonung von Sehakt und Perspektive im Realismus. Das imparfait narratif dokumentiert daher keine Bedeutungsveränderung, vielmehr vermag das imparfait in narrativen Kontexten gerade deshalb einen Spannungseffekt zu erzeugen, weil sein aspektuelles Profil nicht abgeschliffen ist ${ }^{52}$. Das imparfait in narrativen Kontexten ist damit ein (besonders markanter) Verwendungstyp eines unverändert imperfektiven imparfait ${ }^{53}$. Die für die hier unterschiedenen zwei Verwendungsweisen charakteristischen Effekte - einerseits zeitliche Kollision und Kontrastierung, andererseits die Evokation eines perspektivierend wirkenden Wahrnehmungsaktes - entstehen im Zusammenspiel von imperfektiv-meronymischer Semantik und narrativer Kontextorganisation. Beide Sinneffekte sind an spezifische Kontextbedingungen gebunden, sie gehören nicht der einzelsprachlichsystemhaften Bedeutung an, sondern sind ein textsemantisches Phänomen, das auf der Ebene der Texte (und damit der Diskurstraditionen) anzusiedeln ist. Die Funktionsweise des imparfait in narrativen Kontexten erklärt sich damit in einer Synopse, die sowohl das französische Aspektsystem als auch die Diskurstraditionen berücksichtigt. Am Beispiel des „erzählenden“ imparfait wird so das Ineinanderspielen von einzelsprachlichen Traditionen und kulturellen Diskurstraditionen

51 Nach Blumenthal (1986: 102) greift eine systemlinguistische Erklärung, die das imparfait lediglich als Ersatzform für ein sich zurückziehendes passé simple sieht, zu kurz. Vielmehr wird die Ausdehnung der imparfait-Verwendung im 19. Jahrhundert auf eine Technik der „Entlinearisierung“ des Erzählens zurückgeführt (1986: 102 f., 107).

52 In der Forschung wird das imparfait narratif überwiegend als effet de sens und nicht als neuer semantischer Wert des imparfait gesehen. Vgl. hierzu Coseriu (1976: 139), Bres (1999b: bes. 96 f., 112-114, 2005a: 9, 31-49), Caudal / Vetters (2005: 74) und Hunnius (2009: 55). Eine Gegenposition vertritt Gosselin (1999: 25 f., 28 f.) der dem imparfait narratif perfektiven Wert zuordnet. Die Frage, ob das imparfait narratif eine neue imparfait-Semantik konstituiert, ist eng an die Diskussion um die monosemische oder polysemische Semantik des imparfait angebunden, vgl. hierzu die Beiträge in Labeau / Larrivée (Hrsg.) (2005) und Bres (2005a: 13-49).

53 Die Frage nach einer (mit Bedeutungsveränderung verknüpften) Ausdehnung des imparfait muss neben der Verwendung in narrrativ-sukzessiven Kontexten auch weitere Verwendungen wie z. B. Grenzfälle der Iteration berücksichtigen. Vgl. hierzu etwa Togeby (1982: 335-339). 
deutlich. Die Verschränkung beider Wissensbestände belegt, wie eng sprachwissenschaftliche Tempus- und Aspektforschung und literaturwissenschaftliche Erzähltheorie ineinander greifen. Tempora und Aspekte in Texten sind daher in mehrfacher Hinsicht eine „traditionell“ philologische Angelegenheit.

Kassel, im Februar 2012

\section{Bibliographie}

\section{Quellentexte}

Camus, Albert: Les muets, L'hôte, in: L'exil et le royaume, Nachdruck, Paris 1963, Gallimard.

Camus, Albert: La peste, Nachdruck, Paris 1972, Gallimard.

Guide Michelin „Rome“, Michelin et Cie, propriétaires-éditeurs, Clermont-Ferrand 1981.

Guide Michelin „,Corse“, Michelin et Cie, propriétaires-éditeurs, Clermont-Ferrand 1985.

Merlot, André: Précis d'histoire de la littérature française, Paderborn 1960, Librairie Schöningh.

Pagnol, Marcel: La gloire de mon père. Souvenirs d'enfance, Nachdruck, Paris 1988, de Fallois.

Pagnol, Marcel: Le château de ma mère, Nachdruck, Paris 1988, de Fallois.

Simenon, Georges: La Marie du port, Nachdruck, Paris 1975, Gallimard.

Forschungsliteratur

Barbazan, Muriel (2006): Le temps verbal. Dimensions linguistiques et psycholinguistiques, Université de Toulouse-Le Mirail: Presses Universitaires.

Becker, Martin (2010a): „Die Ingredienzen des romanischen Imperfekts“, in: Linguistische Berichte 221, 79-108.

Becker, Martin (2010b): „Passé composé versus passé simple - alles passé?“, in: Romanische Forschungen 122, 3-27.

Berthonneau, Anne-Marie (2000): „L'imparfait de narration dans tous ses états“, in: JeanEmmanuel Tyvaert (Hrsg.), L'imparfait. Philologie électronique et assistance à l'interprétation des textes, Reims: Presses Universitaires, 73-109.

Berthonneau, Anne-Marie / Kleiber, Georges (1993): „Pour une nouvelle approche de l'imparfait: l'imparfait, un temps anaphorique méronomique“, in: Langages 112, $55-73$.

Berthonneau, Anne-Marie / Kleiber, Georges (1999): „Pour une réanalyse de l'imparfait de rupture dans le cadre de l'hypothèse anaphorique et méronomique“, in: Cahiers de praxématique $32,119-166$.

Bertinetto, Pier Marco (1980): „Nuovamente sull'imperfetto narrativo“, in: Lingua nostra 41, 83-89.

Blumenthal, Peter (1986): Vergangenheitstempora, Textstrukturierung und Zeitverständnis in der französischen Sprachgeschichte, Tübingen: Niemeyer.

Bres, Jacques (1999a): „Présentation“, in: Cahiers de praxématique 32, 3-11.

Bres, Jacques (1999b): „L'imparfait dit narratif tel qu'en lui-même (le contexte ne le change pas)“, in: Cahiers de praxématique 32, 87-117.

Bres, Jacques (2005a): L'imparfait dit narratif, Paris: CNRS Éd. 
Bres, Jacques (2005b): „L'imparfait: l'un et / ou le multiple? A propos des imparfaits ,narratif" et d'hypothèse“, in: Emmanuelle Labeau / Pierre Larrivée (Hrsg.), 1-32.

Bres, Jacques (2007): „Sémantique de l'imparfait: dépasser l'aporie de la ,poule“ aspectuelle et de ,l'œuf" anaphorique? Éléments pour avancer", in: Emmanuelle Labeau / Carl Vetters / Patrick Caudal (Hrsg.), Sémantique et diachronie du système verbal français, Amsterdam: Rodopi, 23-46.

Bühler, Karl [1934] (1982): Sprachtheorie. Die Darstellungsfunktion der Sprache, Stuttgart / New York: Gustav Fischer.

Caudal, Patrick / Vetters, Carl (2005): „Que l'imparfait n'est pas (encore) un prétérit“, in: Emmanuelle Labeau / Pierre Larrivée (Hrsg.), 45-77.

Comrie, Bernard (1976): Aspect. An Introduction to the Study of Verbal Aspect and Related Problems, Cambridge u. a.: CUP.

Confais, Jean-Paul (1990): Temps, mode, aspect. Les approches des morphèmes verbaux et leurs problèmes à l'exemple du français et de l'allemand, Toulouse: Presses Universitaires du Mirail.

Coseriu, Eugenio (1976): Das romanische Verbalsystem, herausgegeben und bearbeitet von Hansbert Bertsch, Tübingen: Narr.

Coseriu, Eugenio (1988): Sprachkompetenz. Grundzüge der Theorie des Sprechens, bearbeitet und herausgegeben von Heinrich Weber, Tübingen: Francke.

Coseriu, Eugenio (2000): „Die sprachliche Kompetenz“, in: Bruno Staib (Hrsg.), Linguistica romana et indiana. Festschrift für Wolf Dietrich zum 60. Geburtstag, Tübingen: Narr, 83-97.

Damourette, Jacques / Pichon, Edouard (1936): Des mots à la pensée. Essai de Grammaire de la langue française, vol. 5: Verbe (fin), Paris: d'Artrey.

Fleischman, Suzanne (1982): The Future in Thought and Language. Diachronic Evidence from Romance, Cambridge u. a.: CUP.

García Fernández, Luis (2004): „El pretérito imperfecto: repaso histórico y bibliográfico“, in: Luis García Fernández / Bruno Camus Bergareche (Hrsg.), El pretérito imperfecto, Madrid: Gredos, 13-95.

Gardt, Andreas (2002): „Wort, Text und Bedeutung. Aspekte der semantischen Erschließung von Texten“, in: ders. / Vilmos Ágel / Ulrike Haß-Zumkehr / Thorsten Roelcke (Hrsg.), Das Wort. Seine strukturelle und kulturelle Dimension. Festschrift für Oskar Reichmann zum 65. Geburtstag, Tübingen: Niemeyer, 111-132.

Gardt, Andreas (2008): „Kunst und Sprache. Beobachtungen anlässlich der documenta 12“, in: Achim Barsch / Helmut Scheuer / Georg-Michael Schulz (Hrsg.), Literatur - Kunst Medien. Festschrift für Peter Seibert zum 60. Geburtstag, München: Meidenbauer, 201-224.

Gosselin, Laurent (1999): „Le sinistre Fantomas et l'imparfait narratif“, in: Cahiers de praxématique $32,19-42$.

Hausmann, Franz Josef (1997): „L'imparfait avec et sans mystère“, in: Georges Kleiber / Martin Riegel (Hrsg.), Les formes du sens. Études de linguistique médiévale et générale offertes à Robert Martin à l'occasion de ses 60 ans, Paris: Duculot, 175-180.

Hunnius, Klaus (2009): „Imparfait und Subjonctif. $\mathrm{Zu}$ den Korrespondenzen zwischen ,Tempus' und ,Modus““, in: RJb 60, 51-63.

Imbs, Paul (1960): L'emploi des temps verbaux en français moderne. Essai de grammaire descriptive, Paris: Klincksieck.

Kleiber, Georges (2003): „,Entre les deux mon cœur balance‘ ou l'imparfait entre aspect et anaphore", in: LFr 138, 8-19.

Klum, Arne (1961): Verbe et adverbe. Étude sur le système verbal indicatif et sur le système de certains adverbes de temps à la lumière des relations verbo-adverbiales dans la prose $d u$ français contemporain, Stockholm u. a.: Almqvist \& Wiksell. 
Kött, Martin (2004): Das Interview in der französischen Presse. Geschichte und Gegenwart einer journalistischen Textsorte, Tübingen: Niemeyer.

Labeau, Emmanuelle (2002): „L'unité de l'imparfait: vues théoriques et perspectives pour les apprenants du français langue étrangère“, in: Travaux de linguistique 45, 157-184.

Labeau, Emmanuelle (2005): „Mon nom est narratif: imparfait narratif“, in: dies. / Pierre Larrivée (Hrsg.), 79-102.

Labeau, Emmanuelle (2007): „Et un, ou deux, ou trois? Les temps-champions du reportage sportif depuis 1950“, in: dies. / Carl Vetters / Patrick Caudal (Hrsg.), Sémantique et diachronie du système verbal francais, Amsterdam: Rodopi, 203-233.

Labeau, Emmanuelle / Larrivée, Pierre (Hrsg.) (2005): Nouveaux développements de l'imparfait, Amsterdam: Rodopi.

Maslov, Yurij S. (1985): „An Outline of Contrastive Aspectology“, in: Yurij S. Maslov (Hrsg.), Contrastive Studies in Verbal Aspect, Heidelberg: Groos, 1-44.

Mellet, Sylvie (2000): „Le présent“, in: Travaux de linguistique 40, 97-111.

Mellet, Sylvie (2001): „Valeur aspectuelle du présent: un problème de frontière“, in: Pierre Le Goffic (Hrsg.), Le présent en français, Amsterdam: Rodopi, 27-39.

Mitko, Julia (2000): Aspekt im Französischen. Eine semantisch-funktionelle Analyse, Tübingen: Narr.

Molendijk, Arie (1985): „Point référentiel et imparfait“, in: LFr 67, 78-94.

Muller, Charles (1966): „Pour une étude diachronique de l'imparfait narratif“, in: Mélanges de grammaire française offerts à M. Maurice Grevisse pour le trentième anniversaire du „Bon usage“, Paris: Duculot, 253-269.

Pollak, Wolfgang (21988): Studien zum Verbalaspekt. Mit besonderer Berücksichtigung des Französischen, Bern u. a.: Lang.

Saussure, Louis de (2003): Temps et pertinence. Éléments de pragmatique cognitive du temps, Brüssel: Duculot.

Saussure, Louis de / Sthioul, Bertrand (1999): „L'imparfait narratif: point de vue (et images du monde)“, in: Cahiers de praxématique 32, 167-188.

Saussure, Louis de / Sthioul, Bertrand (2005): „Imparfait et enrichissement pragmatique“, in: Emmanuelle Labeau / Pierre Larrivée (Hrsg.), 103-120.

Schrott, Angela (1997): Futurität im Französischen der Gegenwart. Semantik und Pragmatik der Tempora der Zukunft, Tübingen: Narr.

Schrott, Angela (2003): Rezension zu Mitko (2000), in: RJb 54, 250-257.

Schrott, Angela (2006): Fragen und Antworten in historischen Kontexten. Ein Beitrag zur historischen Dialoganalyse und zur historischen Pragmatik am Beispiel altspanischer literarischer Texte, Habilitationsschrift, Ruhr-Universität Bochum (erscheint im Verlag Klostermann, Frankfurt a. M., in der Reihe Analecta Romanica).

Schrott, Angela (2011): „Von der Lebendigkeit der Heiligenleben. Traditionen der Dialoggestaltung bei Gonzalo de Berceo“, in: Nine Miedema / Monika Unzeitig / Franz Hundsnurscher (Hrsg.), Redeszenen in der mittelalterlichen Großepik: Komparatistische Perspektiven, Berlin: Akademie Verlag, 193-212.

Stempel, Wolf-Dieter (1973): „Erzählung, Beschreibung und der historische Diskurs“, in: Reinhart Koselleck / Wolf-Dieter Stempel (Hrsg.), Geschichte, Ereignis und Erzählung, München: Fink, 325-346.

Tasmowski-de Ryck, Liliane (1985): „L'imparfait avec et sans rupture“, in: LFr 67, $59-77$.

Togeby, Knud (1982): Grammaire française, vol. 2: Les Formes Personnelles du Verbe, publié par Magnus Berg / Ghani Merad / Ebbe Spang-Hanssen, Copenhagen: Akademisk Forlag. 
Vet, Co (1980): Temps, aspects et adverbes de temps en français contemporain. Essai de sémantique formelle, Genf: Droz.

Vet, Co (2005): „L'imparfait: emploi anaphorique et emplois non anaphoriques“, in: Emmanuelle Labeau / Pierre Larrivée (Hrsg.), 33-44.

Vetters, Carl (1996): Temps, aspect et narration, Amsterdam: Rodopi.

Weinrich, Harald ( $\left.{ }^{6} 2001\right):$ Tempus. Besprochene und erzählte Welt, München: Beck. 